\title{
Feminization of the Rheumatology Workforce: A Longitudinal Evaluation of Patient Volumes, Practice Sizes, and Physician Remuneration
}

\author{
Jessica Widdifield ${ }^{1}(\mathbb{D})$, Jodi M. Gatley² (D), Janet E. Pope ${ }^{3}$ (D), Claire E.H. Barber ${ }^{4}$ (D), Bindee Kuriya ${ }^{5}$ (D),

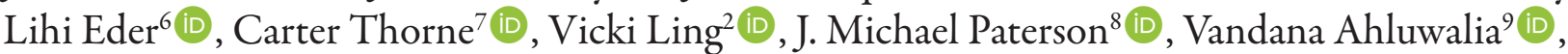 \\ Courtney Marks ${ }^{10}$, and Sasha Bernatsky ${ }^{11}$ (i)
}

\begin{abstract}
Objective. To compare differences in clinical activity and remuneration between male and female rheumatologists and to evaluate associations between physician gender and practice sizes and patient volume, accounting for rheumatologists' age, and calendar year effects.

Methods. We conducted a population-based study in Ontario, Canada, between 2000 to 2015 identifying all rheumatologists practicing as full-time equivalents (FTEs) or above and assessed differences in practice sizes (number of unique patients), practice volumes (number of patient visits), and remuneration (total fee-for-service billings) between male and female rheumatologists. Multivariable linear regression was used to evaluate the effects of gender on practice size and volume separately, accounting for age and year.

Results. The number of rheumatologists practicing at $\geq 1$ FTE increased from 89 to 120 from 2000 to 2015, with the percentage of females increasing from $27.0 \%$ to $41.7 \%$. Males had larger practice sizes and practice volumes. Remuneration was consistently higher for males (median difference of CAD \$46,000-102,000 annually). Our adjusted analyses estimated that in a given year, males saw a mean of 606 (95\% CI 107-1105) more patients than females did, and had 1059 (95\% CI 345-1773) more patient visits. Among males and females combined, there was a small but statistically significant reduction in mean annual number of patient visits, and middle-aged rheumatologists had greater practice sizes and volumes than their younger/older counterparts.

Conclusion. On average, female rheumatologists saw fewer patients and had fewer patient visits annually relative to males, resulting in lower earnings. Increasing feminization necessitates workforce planning to ensure that populations' needs are met.
\end{abstract}

Key Indexing Terms: gender, health human resource planning, rheumatology, workforce

This study was funded by an operating grant from the Canadian Initiative for Outcomes in Rheumatology Care (CIORA), who played no role in the design or conduct of the study, other than providing peer review of the study proposal. This study was supported by ICES, which is funded by an annual grant from the Ontario Ministry of Health and Long-Term Care (MOHLTC). Parts of this material are based on data and information compiled and provided by the MOHLTC. The analyses, conclusions, opinions, and statements expressed herein are solely those of the authors and do not reflect those of the funding or data sources; no endorsement is intended or should be inferred. JW receives support from the Arthritis Society Stars Career Development Award (STAR-19-0610). CEB receives support from the Arthritis Society Stars Career Development Award funded by the Canadian Institutes of Health Research-Institute of Musculoskeletal Health and Arthritis STAR-19-0611/ CIHR SI2-169745. SB holds a career award from the Fonds de la recherche en santé du Québec.

${ }^{1} J$. Widdifield, PhD, ICES, Sunnybrook Research Institute, Holland Bone \& Joint Program, Toronto, and Institute of Health Policy, Management \& Evaluation, University of Toronto, Toronto, Ontario; ${ }^{2} J . M$. Gatley, MPH, V. Ling, MSc, ICES, Toronto, Ontario; ${ }^{3}$. E. Pope, MD, MPH, Division of Rheumatology, Western University, Schulich School of Medicine \& Dentistry, and St Joseph's Health Care, London, Ontario; ${ }^{4}$ C.E. Barber, MD, PhD, The
Cumming School of Medicine, University of Calgary, Calgary, Alberta, and Arthritis Research Canada; ${ }^{5} B$. Kuriya, MD, SM, Division of Rheumatology, University of Toronto, and Sinai Health System, Toronto, Ontario; ${ }^{6}$ L. Eder, $M D, P h D$, Women's College Research Institute, and University of Toronto, Toronto, Ontario; ${ }^{7}$ C. Thorne, $M D$, Department of Medicine, University of Toronto, Toronto, and Southlake Regional Health Centre, Newmarket, Ontario; ${ }^{8}$ J.M. Paterson, MSc, ICES, Toronto, and McMaster University, Department of Family Medicine, Hamilton, and Institute of Health Policy, Management \& Evaluation, University of Toronto, Toronto, Ontario; ${ }^{9} \mathrm{~V}$. Abluwalia, MD, William Osler Health System, Brampton, Ontario; ${ }^{10}$ C. Marks, Sunnybrook Research Institute, Holland Bone \& Joint Program, Toronto, Ontario; ${ }^{\prime \prime}$ S. Bernatsky, MD, PhD, Department of Epidemiology, McGill University, and Research Institute of the McGill University Health Centre, Montreal, Québec, Canada.

The authors declare no conflicts of interest.

Address correspondence to Dr. J. Widdifield, Holland Bone \& Joint Research Program, Sunnybrook Research Institute, MG 352-2075 Bayview Avenue, Toronto, ON M4N 3M5, Canada. Email: jessica.widdifield@utoronto.ca. Open Access Article. For details see Reprints and Permissions at jrheum.org. Accepted for publication November 19, 2020. 
In industrialized countries, there has been a substantial shift in the gender distribution of the medical workforce with physician workforces becoming increasingly female, a concept termed as "feminization of medicine".,2,3 An increasing body of evidence has identified specific attributes of female physicians relative to their male counterparts, including stronger patient-physician partnerships and more effective communication styles, ${ }^{4,5}$ spending more time with their patients, ${ }^{6,7}$ focusing more on preventive health services, ${ }^{4,8,9,10}$ and providing more guideline-concordant care. ${ }^{9,11,12}$ Consequently, patients under the care of female physicians may potentially achieve better patient outcomes, ${ }^{13,14,15}$ although these observations are subject to ongoing debate. Conversely, concerns have been raised that increasing workforce feminization may result in reduced patient access as some studies have found that, on average, female physicians work fewer hours than males, ${ }^{16,17,18,19}$ and/ or work on a part-time basis, ${ }^{19,20}$ and are more likely to take periods of leave. ${ }^{16}$

To date, most attention has been focused on the increasing representation of female physicians in primary care workforces. ${ }^{21}$ However, some specialty-based workforces are increasingly seeing more female representation including urology, ${ }^{22} \mathrm{immu}-$ nology, ${ }^{23}$ dermatology, ${ }^{16}$ geriatric medicine, ${ }^{17}$ endocrinology, ${ }^{18}$ pediatrics, ${ }^{19}$ and obstetrics. ${ }^{20}$ The subspecialty of rheumatology has also seen a restructuring of the gender composition of its workforce. In Canada, females accounted for less than one-third of rheumatologists in 1995, but reached parity in 2015, with an upward trajectory since. ${ }^{24}$ In particular, many younger Canadian rheumatologists are female, accounting for $72 \%$ of rheumatologists $<45$ years of age as of $2018 .{ }^{24}$ Limited research in this field suggests that female rheumatologists may also have different practice patterns than males. ${ }^{25,26,27}$ While less is known about gender differences in practice patterns specific to the rheumatology setting, the increasing proportion of females in the rheumatology workforce may have implications for future physician supply and patient access.

In light of the rapidly changing demographics of the rheumatology workforce, it is crucial to understand how feminization is influencing clinical capacity. Thus, we sought to compare differences in clinical activity and remuneration between male and female rheumatologists in a government-funded healthcare setting, and to evaluate the effects of physician gender on clinical activity, accounting for rheumatologists' age and calendar year effects.

\section{METHODS}

Study design and setting. We conducted a population-based, retrospective, observational study in Ontario, Canada. We analyzed the administrative databases held at ICES from the province of Ontario, comprising data of approximately 14 million residents (as of 2015) who are covered under a publicly funded healthcare system. As of 2015 , approximately $40 \%$ of the Canadian rheumatology workforce resided in Ontario. ${ }^{26}$

Data sources. We identified rheumatologists and patient visits with rheumatologists between April 1, 2000 and March 31, 2015. Rheumatologists were identified using the ICES Physician Database, a physician registry that is constructed and regularly validated using the Ontario Health Insurance Plan (OHIP) Corporate Provider Database, the Ontario Physician Human
Resources Data Centre Database, and physicians' OHIP fee-for-service billing claims. The ICES Physician Database provides information on physician demographics and types of certified specialties, and is linkable to the OHIP Claims History Database to quantify the health services provided by each physician (detailed below). Rheumatologists were excluded from years where they had no claims, with the date of the last claim used to identify the year first excluded. They could reenter the workforce upon resuming activity.

The OHIP Claims History Database was used to identify patients $\geq 18$ years of age seen by rheumatologists. The services rendered are identified using specific fee codes in the OHIP Schedule of Benefits. These billing claims also indicate the location of service (e.g., hospital vs office based). ${ }^{28}$ We obtained patient demographic information and vital status from the OHIP Registered Persons Database.

These datasets were linked using unique encoded identifiers and analyzed at ICES. (www.ices.on.ca). The use of the data in this project was authorized under section 45 of Ontario's Personal Health Information Protection Act, which does not require review by a research ethics board.

Analysis. All outcomes were reported at the provider level and were stratified by physician gender and aggregated on an annual basis. In order to quantify clinical activity, we calculated clinical full-time equivalents (FTEs) using annual fee-for-service billing claims, which are submitted by all rheumatologists in Ontario including those working in hospitals. Rheumatologists practicing at least 1 clinical FTE were defined as those who were at or above the 40th percentile of total billings each year. ${ }^{29}$ We identified the total number of rheumatologists and the number working at least 1 clinical FTE. Rheumatologists practicing $<1$ FTE (about one-third of the Ontario rheumatology workforce during this period $)^{25}$ were not included in the remaining analyses of practice sizes, volumes, and remuneration as their primary roles are more likely to be in research, administration, and/ or teaching, rather than clinical care and they therefore also receive institutional salary support outside of fee-for-service billing claims.

We also identified rheumatologists who worked at least 209 days in a given year (an alternative FTE benchmark) ${ }^{25}$ with at least 3 patient visits on those days, as a secondary measure of clinical activity in order to account for number of days spent providing clinical care. Patient contacts were defined as any in-person clinical service (i.e., patient claim) with any outpatient or inpatient fee code.

Annual median practice volume (i.e., the number of patient visits in an outpatient setting) and median practice size (i.e., the number of unique patients seen) were reported. We also described the number of rheumatologists according to practice size and practice volume categories in 2000, 2007, and 2015. Practice size categories were based on the number of unique patients seen (i.e., < 1500, 1500-1999, or $\geq 2000$ patients), whereas practice volume categories were based on the number of patient visits (i.e., < 3500, 3500-4999, or $\geq 5000$ patient visits). We estimated median payments to rheumatologists in Canadian dollars, defined as the sum of paid fee-for-service billings and alternative payments (e.g., from additional hospital service). Median was chosen for outcomes with nonnormal distributions. For all outcomes, effect sizes were reported as either mean or median differences. We compared annual values by gender each year using either the Mann-Whitney $U$ test or chi-square test, as appropriate.

Multivariable linear regression was used to evaluate the effect of physician gender on practice volume and practice size separately, controlling for physician age (categorical) and year (continuous). We tested an interaction between gender and year by running models separately by gender for the effect of individual years on practice size and practice volume, controlling for physician age. In a sensitivity analysis, rheumatologists who fell within the highest and lowest $5 \%$ of practice volume or practice size were excluded, and all models were run again with the truncated sample. This was intended to evaluate whether outlier physicians influenced the results.

All analyses were performed at ICES using SAS version 7.15 (SAS Institute).

Personal non-commercial use only. The Journal of Rheumatology Copyright (c) 2021. All rights reserved

Widdifield, et al: Feminization of rheumatology 


\section{RESULTS}

Annual total number of all rheumatologists and clinical FTEs by physician gender. The total number of rheumatologists increased from 146 in 2000 to 194 in 2015 (Table 1). Overall, there was an increasing percentage of females in the workforce over time, reaching $49.0 \%$ of the workforce in 2015 .

Across all years, the percentage of rheumatologists working at least 1 clinical FTE ranged from $25.3 \%$ to $43.0 \%$ for females, whereas $57.0 \%$ to $74.7 \%$ of the clinical FTE workforce was male over the same time period. Approximately half of all female rheumatologists worked in a clinical FTE capacity, whereas the majority (64.7-72.0\%) of males were working in an FTE capacity during this period (Table 1).

As an alternative clinical FTE measure, the total number of rheumatologists who saw at least 3 patients in any setting on at least 209 days out of the year identified 15-20 females (40-72\%) and 43-55 males (67-85\%) annually (Supplementary Table 1, available with the online version of this article). A significantly lower percentage of females met this secondary benchmark of clinical activity, as compared to males, across over two-thirds of the study years.

Comparison of practice sizes and volumes between female and male rheumatologists. Among both male and female clinical FTE rheumatologists, there were trends toward decreasing median practice sizes (number of unique patients seen annually) over the study period (Table 2). For females, median practice size was 1605.5 patients (IQR 1276-2228) in 2000 vs 1468.5 patients (IQR 1212-1984) in 2015. For males, median practice size was 2242 patients (IQR 1606-2936) in 2000 vs 1948.5 patients (IQR 1433-2562) in 2015. Males had significantly larger median practice sizes compared to females in all but 1 year (2001). The gap in median practice sizes between males and females fluctuated over the study span, with a nonsignificant gap of 356 (95\% CI -92 to $805, P=0.11$ ) in 2001, up to a high of 677 more patients for males (95\% CI 389-979, $P<0.0001$ ) in 2012.

While practice sizes declined over time for both genders, median practice volumes (total patient visits annually) were relatively stable for males, but trended toward decline for females over the study span, with the gap widening over time. Males had significantly larger median practice volumes in most years. Gaps in median practice volume between males and females varied over the study period, ranging from a minimum nonsignificant gap of 668 visits in 2001 (95\% CI -73 to 1490) to a peak of 1486 more patient visits (95\% CI 628-2517) for male rheumatologists in 2008 (Table 2).

When median practice size was examined categorically over 3 years $(2000,2007$, and 2015), there was a consistently smaller percentage of female rheumatologists in the "large" practice category (i.e., $\geq 2000$ unique patients) and in the highest practice volume category (i.e., $\geq 5000$ patient visits) relative to male rheumatologists (Supplementary Table 2, available with the online version of this article).

Comparison of annual remuneration between female and male rheumatologists. The annual median payments to individual rheumatologists increased for both males and females during the study period (Table 3 ). Median female remuneration spanned $\$ 199,730$ (IQR \$180,696-262,409) in 2000 to \$362,522 (IQR $\$ 309,503-437,127)$ by 2015 , whereas male remuneration went from $\$ 285,930$ (IQR $\$ 211,796-354,507$ ) in 2000 to $\$ 403,903$

Table 1. Annual total number of all rheumatologists and rheumatologists working at least 1 clinical FTE in Ontario, between 2000 and 2015 , by physician gender.

Rheumatologists

Working At Least 1

FTE Out of the Total

No. Rheumatologists

Within Each Gender

\begin{tabular}{|c|c|c|c|c|c|c|c|c|}
\hline \multirow[b]{2}{*}{ Year } & \multirow[b]{2}{*}{ Total, $\mathrm{n}$} & \multirow[b]{2}{*}{ Female, n (\%) } & \multirow[b]{2}{*}{ Male, n (\%) } & \multirow[b]{2}{*}{ Total, $\mathrm{n}$} & \multirow[b]{2}{*}{ Female, n (\%) } & \multirow[b]{2}{*}{ Male, n (\%) } & \\
\hline & & & & & & & Female, \% & Male, \% \\
\hline 2000 & 146 & $50(34.2)$ & $96(65.8)$ & 89 & $24(27.0)$ & $65(73.0)$ & 48.0 & 67.7 \\
\hline 2001 & 152 & $51(33.6)$ & $101(66.4)$ & 92 & $25(27.2)$ & $67(72.8)$ & 49.0 & 66.3 \\
\hline 2002 & 154 & $51(33.1)$ & $103(66.9)$ & 95 & $24(25.3)$ & $71(74.7)$ & 47.1 & 68.9 \\
\hline 2003 & 148 & $53(35.8)$ & $95(64.2)$ & 91 & $26(28.6)$ & $65(71.4)$ & 49.1 & 68.4 \\
\hline 2004 & 148 & $53(35.9)$ & $95(64.2)$ & 90 & $24(26.7)$ & $66(73.3)$ & 45.3 & 69.5 \\
\hline 2005 & 151 & $54(35.8)$ & $97(64.2)$ & 91 & $26(28.6)$ & $65(71.4)$ & 48.2 & 67.0 \\
\hline 2006 & 154 & $55(35.7)$ & $99(64.3)$ & 94 & $30(31.9)$ & $64(68.1)$ & 54.6 & 64.7 \\
\hline 2007 & 155 & $59(38.1)$ & $96(61.9)$ & 94 & $29(30.9)$ & $65(69.2)$ & 49.2 & 67.7 \\
\hline 2008 & 156 & $62(39.7)$ & $94(60.3)$ & 96 & $35(36.5)$ & $61(63.5)$ & 56.5 & 64.9 \\
\hline 2009 & 160 & $68(42.5)$ & $92(57.5)$ & 97 & $31(32.0)$ & $66(68.0)$ & 45.6 & 71.7 \\
\hline 2010 & 161 & $68(42.2)$ & $93(57.8)$ & 98 & $31(31.6)$ & $67(68.4)$ & 45.6 & 72.0 \\
\hline 2011 & 176 & $79(44.9)$ & $97(55.1)$ & 107 & $39(36.5)$ & $68(63.6)$ & 49.4 & 70.1 \\
\hline 2012 & 177 & $81(45.8)$ & $96(54.2)$ & 107 & $43(40.2)$ & $64(59.8)$ & 53.1 & 66.7 \\
\hline 2013 & 186 & $89(47.8)$ & $97(52.2)$ & 114 & $49(43.0)$ & $65(57.0)$ & 55.1 & 67.0 \\
\hline 2014 & 183 & $89(48.6)$ & $94(51.4)$ & 114 & $48(42.1)$ & $66(57.9)$ & 53.9 & 70.2 \\
\hline 2015 & 194 & $95(49.0)$ & $99(51.0)$ & 120 & $50(41.7)$ & $70(58.3)$ & 52.6 & 70.7 \\
\hline
\end{tabular}

${ }^{a}$ Denominator for $\%$ is the total of FTE rheumatologists. FTE: full-time equivalent. 


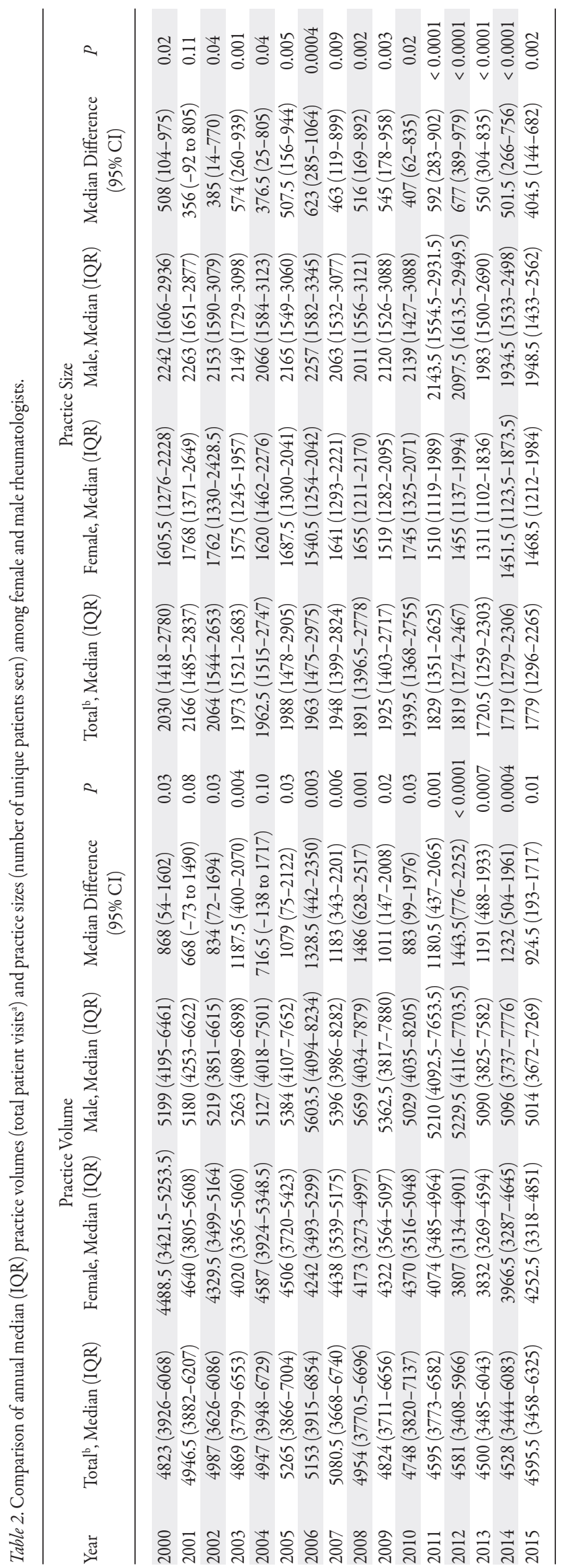

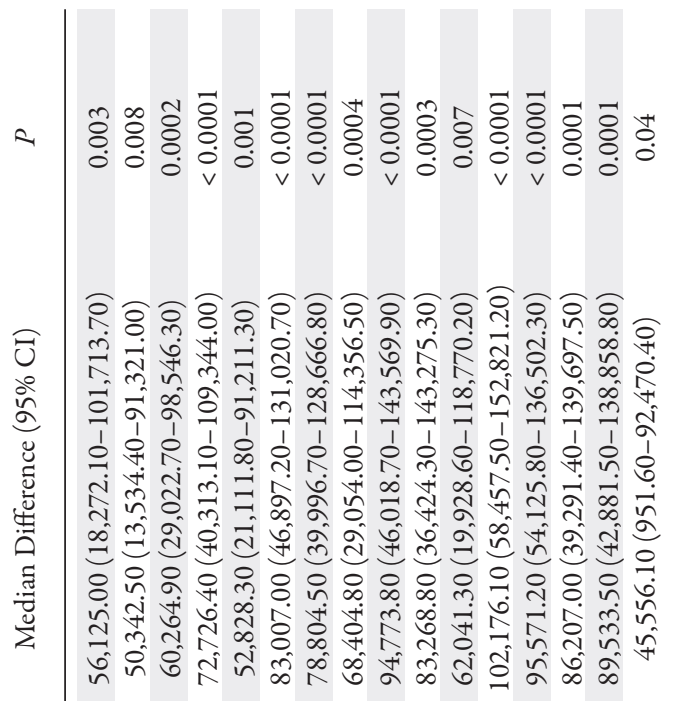

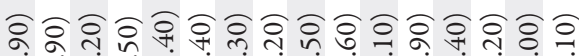

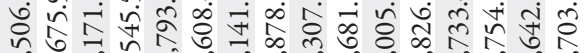

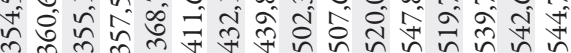

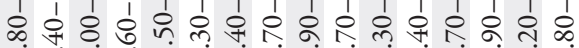

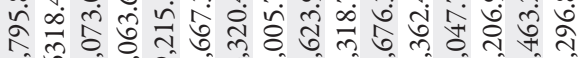

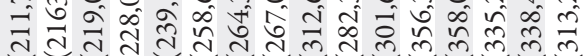

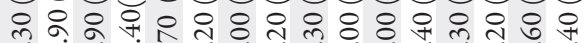

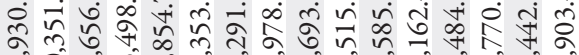

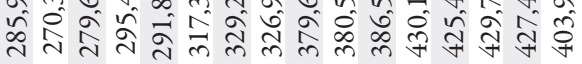

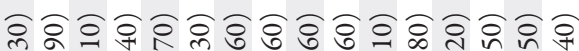

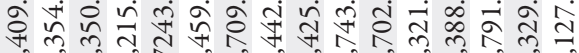
ज़่

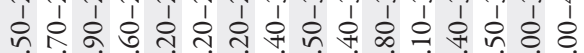

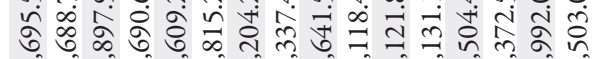
D.

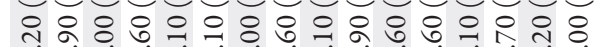

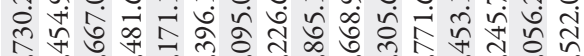

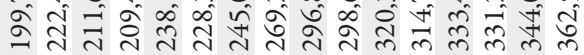

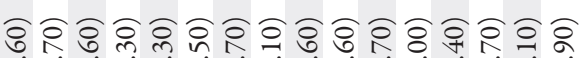

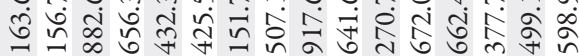

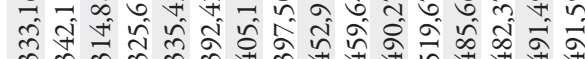
1́

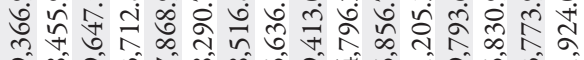
ڤे

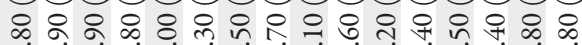

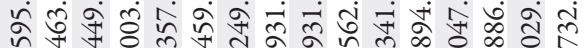

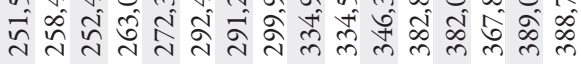

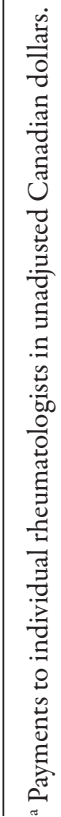


(IQR \$313,297-544,703) in 2015. Across years, male remuneration was significantly higher than female remuneration, with the median difference (gross earnings gap) ranging from $\$ 45,556$ to $\$ 102,176$.

Association of rheumatologist gender with practice volume and sizes. In the multivariable regression model for practice volume (adjusted for age and year), we found that female gender was associated with lower practice volume $(-1058.9$ patient visits, $95 \%$ CI -1773.3 to -344.5 ; Table 4 ). In the model for gender and practice size (adjusted for age and year), there was also an association with female gender and lower practice size $(-605.8$ unique patients, 95\% CI -1104.5 to -107.1). Rheumatologists in the middle age group (45-64 yrs of age) tended to have larger practice sizes and service volumes, whereas older providers $(\geq 65$ yrs of age) tended to have lower service volumes, as compared to the younger rheumatologists ( $<45$ yrs of age), adjusting for gender and year.

In the multivariable regression models stratified by gender, there was an increase in practice volume among male rheumatologists in 2008 of 1458.7 visits (95\% CI 82.3-2835.0), relative to 2000, and adjusted for age (Supplementary Table 3, available with the online version of this article). Practice volume declined among males $\geq 65$ years of age (by -1254.3 visits annually, $95 \%$ CI -2145.0 to -363.6 ), whereas practice size increased among middle-aged males 45-64 years of age (by 1028.7 patients annually, 95\% CI 639.4-1417.9), both relative to the youngest age group (< 45 yrs of age). Among females, practice volume increased for middle-aged women by 498.6 visits annually $(95 \%$ CI 267.0-730.3).

In the sensitivity analyses involving the truncated sample, our multivariable regression model confirmed that female gender was still negatively associated with both practice size and practice volume after adjusting for age and year (Supplementary Table 4, available with the online version of this article). However, increase in year was negatively associated with practice size (fewer unique patients being seen annually), with no effect for practice volume. Relationships with physician age were similar to those in the main analysis, except that there was no effect of belonging to the middle age group on practice volume. In the models for year stratified by gender and adjusted for age, there were no clear effects within genders on practice volume, but practice sizes fluctuated over time (Supplementary Table 5, available with the online version of this article). Similar results to those observed in the main analyses were seen for age group in the gender-stratified models, controlling for year.

\section{DISCUSSION}

We examined longitudinal changes in clinical activity and remuneration between male and female rheumatologists over a 15-year period in the setting of a provincial-payer healthcare system for all rheumatology services. We observed growth in the number of rheumatologists and those working at least 1 clinical FTE. The percentage of rheumatologists who worked at least 1 clinical FTE was consistently higher for males (65-72\%) than females (50\%). By calendar year, median practice sizes tended to decline for both genders, whereas practice volumes were relatively stable for males but declining among females. Female rheumatologists nearly always had lower practice sizes, patient volumes, and remuneration than males. After controlling for age and year, female gender was still associated with lower practice sizes and practice volumes. These findings highlight key differences in volume of services between males and females that are essential to account for in rheumatology workforce planning.

Our findings of decreasing practice size and practice volume for both female and male rheumatologists in Ontario are in line with prior findings within rheumatology, for otherclinical specialties, and among physicians, more generally, in Canada. $25,30,31,32,33$ Previously, we found that the median number of days with any patient visit fell from 231 days to 205.5 days between 2000 and 2015 among all rheumatologists in Ontario..$^{25} \mathrm{~A}$ separate study of all physicians in Ontario found a $14 \%$ decrease in yearly patient visits from 1992 to 2013, although the number of distinct patients seen was stable. ${ }^{32}$ In Canada, decreases in weekly work hours devoted to patient care have been observed among all physicians since the early $1990 s^{30,31,32,33}$ These reductions may reflect the trend among younger cohorts of physicians to have decreasing numbers of visits each year compared to physicians from older generations. Indeed, younger physicians ( $<35$ yrs of age) in the United States and Canada report working fewer hours than previous cohorts, ${ }^{34,35}$ which may partly reflect the high importance placed on work-life balance among this generational group. Additional contributing factors could be the increasing

Table 4. Multivariable linear regression models testing the association of rheumatologist gender with mean practice volume (total patient visits) and annual mean practice size (number of unique patients seen) among rheumatologists between 2000 and 2015.

\begin{tabular}{|c|c|c|c|c|}
\hline \multicolumn{5}{|l|}{ Variables } \\
\hline \multicolumn{5}{|l|}{ Gender } \\
\hline Female (ref: male) & -1058.9 & $(-1773.3$ to -344.5$)$ & -605.8 & $(-1104.5$ to -107.1$)$ \\
\hline $45-64$ & 385.9 & $(26.3-745.5)$ & 620.1 & $(369.1-871.2)$ \\
\hline$\geq 65$ & -1144.8 & $(-1841.8$ to -447.7$)$ & -27.3 & $(-513.9$ to 459.3$)$ \\
\hline Interaction term, yr and female gender & -114.9 & $(-189.7$ to -40.2$)$ & -57.8 & $(-110$ to -5.6$)$ \\
\hline
\end{tabular}


complexity of care, structural changes in care delivery (e.g., electronic medical records [EMR] and more nonpatient work such as form completion), or more external consulting activities. For example, an observational study of American physicians across various specialties found nearly 2 hours were spent on administrative work and EMR completion for each hour of face-to-face time with patients. ${ }^{36}$

A central concern of these findings is that since female rheumatologists see fewer patients, increased feminization of the rheumatology workforce may lead to longer patient wait-times and reduced clinical capacity. Many barriers may be preventing females from working at the same clinical capacity as their male peers. Prior research suggests that gender differences in work patterns among physicians are strongly tied to family characteristics including relationship status and children, and may be influenced by societal expectations in domestic and caregiving responsibilities. ${ }^{30,31,37}$ Female physicians are also more likely to take periods of leave for parental leave, illness or injury, or other reasons, ${ }^{37}$ yet service capacity appears to rebound to a higher level following child-bearing years, before declining with older age, similar to male physicians. ${ }^{31}$ The prevalence of burnout among female physicians is $20 \%$ to $60 \%$ higher than among males, and there is evidence that this is influenced by societal expectations placing a disproportionate burden on female physicians. ${ }^{38}$ Workforce disparities for women in rheumatology almost certainly play some role in physician burnout. Many female physicians also report encountering sex- or gender-based discrimination in the workplace, and fewer females are represented in leadership and mentor positions. ${ }^{38,39,40,41}$ On the other hand, the higher clinical activity of male rheumatologists may indicate that men are more likely to be the primary income earner in their household, ${ }^{42}$ placing higher demands on them to see more patients. Additionally, males are more likely to be older physicians with established practices, and may receive more referrals than their female peers.

Our finding of year-over-year increases in remuneration is also in line with increases in reimbursement fees for services to Ontario physicians implemented over the study period. ${ }^{43}$ As both male and female Ontario rheumatologists are paid the same flat rates for services regardless of age, gender, or other physician characteristics (which in theory ensures that males and females receive the same remuneration for the same service), gender differences in remuneration can be assumed to reflect differences in practice patterns. However, it should be noted that payments to rheumatologists in our study do not directly reflect the net incomes rheumatologists receive as they are not adjusted for overhead and other expenses incurred by rheumatologists in running their practices. In Ontario, there is no specific funding allocated for administrative overhead, nursing, physiotherapists, occupational therapists, nurse practitioners, and physician assistants, which are essential to supporting rheumatology practices. Overhead is likely to vary substantially by practice, and it is difficult to construct a complete account of rheumatologists' incomes. ${ }^{44}$

It is important to consider that differing practice patterns in male vs female specialists may have complex implications for health system sustainability, costs, and quality of care, in a time when the burden of rheumatic and musculoskeletal diseases is increasing with population aging and expansion (i.e., immigration)..$^{45}$ Health human resources planning needs to consider workforce feminization to offset potential negative consequences of lower supply. For instance, it may require more than 1 rheumatologist to take the place of an established male rheumatologist exiting the workforce. To inform policies and strategies related to improving supply of rheumatologists, future research is needed to predict the future rheumatology supply requirements and demand for rheumatology care, taking into account population needs as well as changing workforce clinical capacity. While the true deficit in rheumatology supply has not fully been quantified, immediate efforts to expand recruitment of clinical FTEs are needed, considering the lag time required to train new rheumatologists. Moreover, more research is needed to better understand the distribution of patient care, potential differences in practice patterns and clinical case mix, and quality of care between male and female rheumatologists.

A key strength of this study is the use of population-based data from a large publicly funded healthcare system, which captured virtually all rheumatology billing claims and physician demographics in a province of 14 million residents (as of 2015). To our knowledge, this is the first Canadian study to directly compare differences in clinical activity and remuneration between male and female rheumatologists. Ontario, being the most populous Canadian province, contains the largest Canadian rheumatology workforce, and while rheumatology supply differs across Canada, changing workforce characteristics in Ontario are likely to be generalizable to the broader Canadian and international rheumatology workforces. However, the effects of increasing workforce feminization may have larger implications in settings with smaller rheumatology workforces where access to rheumatology care is already strained.

Despite these strengths, we acknowledge potential limitations. There is no standard definition of a clinical FTE rheumatologist and there are no records available of exact hours worked by physicians in Ontario. We chose to define FTEs by setting a percentile cutoff of fee-for-service billings as a proxy measure, as has been used previously, and which is not affected by changes in fee levels over time. ${ }^{29}$ In this study, the 40 th percentile of billings was used to define $1 \mathrm{FTE}$; simulations demonstrate that FTE counts are generally insensitive across benchmark ranges (e.g., 60 th vs 70 th percentile) to define high-volume providers. In light of the lack of consensus on defining FTE, we also presented an alternative measure based on the annual number of days providing clinical care. ${ }^{25}$ Further, we do not have reasons as to why certain rheumatologists were practicing less than a clinical FTE. We assumed their primary responsibilities were outside of providing clinical care (e.g., research, administrative, and teaching); however, they may have also opted to work part time.

Another limitation is that we could only focus on gender, and did not have access to information about gender-related factors (e.g., marital status, family size, and childcare roles) that could contribute to the relationship between physician gender and practice-level activity. Additionally, we lacked information 
on whether or not rheumatologists were practicing in multidisciplinary care teams and we were unable to assess differences between academic and community-based practices.

In summary, the current study provides crucial information about gender differences in clinical activity and practice characteristics of rheumatologists. In order for future health workforce policy and planning to be effective and equitable, it is essential to consider policies and other solutions to support the sustainability of rheumatology workforces in light of increasing feminization, particularly in an era of increasing demand for rheumatology services and an already strained rheumatology supply. Future research should aim to further delineate possible gender differences in practice patterns and quality of care.

\section{ONLINE SUPPLEMENT}

Supplementary material accompanies the online version of this article.

\section{REFERENCES}

1. The Association of Faculties of Medicine of Canada. Canadian medical education statistics 2018 Ottawa: AFMC; 2019.

2. Young A, Chaudhry HJ, Pei X, Arnhart K, Dugan M, Snyder GB. A census of actively licensed physicians in the United States, 2016. J Med Regul 2017;103:7-21.

3. General Medical Council. The state of medical education and practice in the UK 2017. London: General Medical Council; 2017.

4. Bertakis KD, Franks P, Azari R. Effects of physician gender on patient satisfaction. J Am Med Womens Assoc 2003;58:69-75.

5. Roter DL, Hall JA, Aoki Y. Physician gender effects in medical communication: a meta-analytic review. JAMA 2002;288:756-64.

6. French F, Andrew J, Awramenko M, Coutts H, Leighton-Beck L, Mollison J, et al. Why do work patterns differ between men and women GPs? J Health Organ Manag 2006;20:163-72.

7. Franks P, Bertakis KD. Physician gender, patient gender, and primary care. J Womens Health 2003;12:73-80.

8. Frank E, Harvey LK. Prevention advice rates of women and men physicians. Arch Fam Med 1996;5:215-9.

9. Henderson JT, Weisman CS. Physician gender effects on preventive screening and counseling: an analysis of male and female patients' health care experiences. Med Care 2001;39:1281-92.

10. Baig AA, Heisler M. The influence of patient race and socioeconomic status and resident physician gender and specialty on preventive screening. Semin Med Pract 2008;11:27-35.

11. Baumhäkel M, Müller U, Böhm M. Influence of gender of physicians and patients on guideline-recommended treatment of chronic heart failure in a cross-sectional study. Eur J Heart Fail 2009;11:299-303.

12. Berthold HK, Gouni-Berthold I, Bestehorn KP, Böhm M, Krone W. Physician gender is associated with the quality of type 2 diabetes care. J Intern Med 2008;264:340-50.

13. Dahrouge S, Seale E, Hogg W, Russell G, Younger J, Muggah E, et al. A comprehensive assessment of family physician gender and quality of care: a cross-sectional analysis in Ontario, Canada. Med Care 2016;54:277-86.

14. Tsugawa Y, Jena AB, Figueroa JF, Orav EJ, Blumenthal DM, Jha AK. Comparison of hospital mortality and readmission rates for Medicare patients treated by male vs female physicians. JAMA Intern Med 2017;177:206-13.

15. Wallis CJ, Ravi B, Coburn N, Nam RK, Detsky AS, Satkunasivam R. Comparison of postoperative outcomes among patients treated by male and female surgeons: a population based matched cohort study. BMJ 2017;359:j4366.

16. Bae G, Qiu M, Reese E, Nambudiri V, Huang S. Changes in sex and ethnic diversity in dermatology residents over multiple decades. JAMA Dermatol 2016;152:92-4.

17. Fisher JM, Garside M, Hunt K, Lo N. Geriatric medicine workforce planning: a giant geriatric problem or has the tide turned? Clin Med 2014; 14:102-6.

18. Pelley E, Danoff A, Cooper DS, Becker C. Female physicians and the future of endocrinology. J Clin Endocrinol Metab 2016; 101:16-22.

19. Spector ND, Cull W, Daniels SR, Gilhooly J, Hall J, Horn I, et al. Gender and generational influences on the pediatric workforce and practice. Pediatrics 2014;133:1112-21.

20. Pearse WH, Haffner WH, Primack A. Effect of gender on the obstetric-gynecologic work force. Obstet Gynecol 2001;97:794-7.

21. Jefferson L, Bloor K, Maynard A. Women in medicine: historical perspectives and recent trends. Br Med Bull 2015;114:5-15.

22. Nam CS, Daignault-Newton S, Herrel LA, Kraft KH. The future is female: urology workforce projection from 2020 to 2060. Urology 2020 Sep 6 (in press).

23. Marshall GD, American Academy of Allergy, Asthma \& Immunology Workforce Committee. The status of US allergy/ immunology physicians in the 21 st century: a report from the American Academy of Allergy, Asthma \& Immunology Workforce Committee. J Allergy Clin Immunol 2007;119:802-7.

24. Canadian Medical Association. Rheumatology profile. Ottawa: CMA; 2018. [Internet. Accessed March 11, 2021.] Available from: www.cma.ca/canadian-specialty-profiles

25. Widdifield J, Bernatsky S, Pope JE, Ahluwalia V, Barber CE, Eder $\mathrm{L}$, et al. Encounters with rheumatologists in a publicly-funded Canadian healthcare system: a population-based study. J Rheumatol 2020;47:468-76.

26. Barber CE, Jewett L, Badley EM, Lacaille D, Cividino A, Ahluwalia V, et al. Stand up and be counted: measuring and mapping the rheumatology workforce in Canada. J Rheumatol 2017;44:248-57.

27. Barber CE, Nasr M, Barnabe C, Badley EM, Lacaille D, Pope J, et al. Planning for the rheumatologist workforce: factors associated with work hours and volumes. J Clin Rheumatol 2019;25:142-6.

28. Government of Ontario, Ministry of Health and Long Term Care. Ontario health insurance plan schedule of benefits and fees. Updated 2019. [Internet. Accessed February 18, 2021.] Available from: health.gov.on.ca/en/pro/programs/ohip/sob

29. Canadian Institute for Health Information. National physician database data release, 2015-2016: Methodology notes. [Internet. Accessed March 11, 2021.] Available from: www.cihi.ca/sites/ default/files/document/national-physician-database-data-release2018-2019-methodology-notes-en.pdf

30. Wang C, Sweetman A. Gender, family status and physician labour supply. Soc Sci Med 2013;94:17-25.

31. Sarma $S$, Thind A, Chu MK. Do new cohorts of family physicians work less compared to their older predecessors? The evidence from Canada. Soc Sci Med 2011;72:2049-58.

32. Buys YM, Canizares M, Felfeli T, Jin Y. Influence of age, sex, and generation on physician payments and clinical activity in Ontario, Canada: an age-period-cohort analysis. Am J Ophthalmol 2019;197:23-35.

33. Watson DE, Slade S, Buske L, Tepper J. Intergenerational differences in workloads among primary care physicians: a ten-year, population-based study. Health Aff 2006;25:1620-8.

34. Deal CL, Hooker R, Harrington T, Birnbaum N, Hogan P, Bouchery E, et al. The United States rheumatology workforce: supply and demand, 2005-2025. Arthritis Rheum 2007;56:722-9.

35. The College of Family Physicians of Canada, Canadian Medical Association, Royal College of Physicians and Surgeons of Canada. 2014 national physician survey: national results by FP/GP or other specialist, sex, age and all physicians [Internet. Accessed February 
18, 2021.] Available from: nationalphysiciansurvey.ca/wp-content/ uploads/2014/11/2014-National-EN.pdf

36. Sinsky C, Colligan L, Li L, Prgomet M, Reynolds S, Goeders L, et al. Allocation of physician time in ambulatory practice: a time and motion study in 4 specialties. Ann Intern Med 2016;165:753-60.

37. Weizblit N, Noble J, Baerlocher MO. The feminisation of Canadian medicine and its impact upon doctor productivity. Med Educ 2009; 43:442-8.

38. Templeton KC, Bernstein CA, Sukhera J, Nora LM, Newman $\mathrm{C}$, Burstin $\mathrm{H}$, et al. Gender-based differences in burnout: issues faced by women physicians. NAM Perspectives. Discussion Paper, National Academy of Medicine 2019. Available from: nam.edu/ gender-based-differences-in-burnout-issues-faced-by-womenphysicians. doi.org/10.31478/201905a

39. Butkus R, Serchen J, Moyer DV, Bornstein SS, Hingle ST, Health and Public Policy Committee of the American College of Physicians. Achieving gender equity in physician compensation and career advancement: A position paper of the American College of Physicians. Ann Intern Med 2018;168:721-3.

40. Mamtani M, Shofer F, Mudan A, Khatri U, Walker R, Perrone J, et al. Quantifying gender disparity in physician authorship among commentary articles in three high-impact medical journals: an observational study. BMJ Open 2020;10:e034056.
41. Lee D, Jalal S, Nasrullah M, Ding J, Sanelli P, Khosa F. Gender disparity in academic rank and productivity among public health physician faculty in North America. Cureus 2020;12:e8553.

42. Jolly S, Griffith KA, DeCastro R, Stewart A, Ubel P, Jagsi R. Gender differences in time spent on parenting and domestic responsibilities by high-achieving young physician-researchers. Ann Intern Med 2014; 160:344-53.

43. Henry DA, Schultz SE, Glazier RH, Bhatia RS, Dhalla IA, Laupacis A. Payments to ontario physicians from Ministry of Health and Long-Term Care sources 1992/93 to 2009/10. ICES investigative report. [Internet. Accessed March 11, 2021.] Available from: https://www.ices.on.ca/publications/atlases-and-reports/2012/ payments-to-ontario-physicians

44. Petch J, Dhalla IA, Henry DA, Schultz SE, Glazier RH, Bhatia S, et al. Public payments to physicians in Ontario adjusted for overhead costs. Healthc Policy 2012;8:30-6.

45. Al Maini M, Adelowo F, Al Saleh J, Al Weshahi Y, Burmester GR, Cutolo M, et al. The global challenges and opportunities in the practice of rheumatology: white paper by the World Forum on Rheumatic and Musculoskeletal Diseases. Clin Rheumatol 2015;34:819-29. 